\title{
Robust production of uniform human cerebral organoids from pluripotent stem cells
}

\author{
Adam A Sivitilli, ${ }^{1,2}$, Jessica T Gosio ${ }^{3,4}$, Bibaswan Ghoshal ${ }^{4}$, Alesya Evstratova ${ }^{5}$, Daniel Trcka ${ }^{3,4}$, Parisa Ghiasi, ${ }^{1,2}$, \\ J Javier Hernandez ${ }^{3,4}$, Jean Martin Beaulieu ${ }^{5}$, Jeffrey L Wrana ${ }^{3,4}$, Liliana Attisano ${ }^{1,2}$ (1)
}

\begin{abstract}
Human cerebral organoid (hCO) models offer the opportunity to understand fundamental processes underlying human-specific cortical development and pathophysiology in an experimentally tractable system. Although diverse methods to generate brain organoids have been developed, a major challenge has been the production of organoids with reproducible cell type heterogeneity and macroscopic morphology. Here, we have directly addressed this problem by establishing a robust production pipeline to generate morphologically consistent hCOs and achieve a success rate of $>80 \%$. These hCOs include both a radial glial stem cell compartment and electrophysiologically competent mature neurons. Moreover, we show using immunofluorescence microscopy and single-cell profiling that individual organoids display reproducible cell type compositions that are conserved upon extended culture. We expect that application of this method will provide new insights into brain development and disease processes.
\end{abstract}

DOI 10.26508/lsa.202000707 | Received 18 March 2020 | Revised 3 April 2020 | Accepted 6 April 2020 | Published online 17 April 2020

\section{Introduction}

The development, patterning, and homeostatic maintenance of the human brain is complex and while considerable insights into mechanisms driving these processes have been obtained from studies in model organisms, species-specific differences in brain development and function can make it challenging to apply results from animal models to humans. Accordingly, understanding the molecular basis underlying normal development, disease progression, and therapeutic options for human brain-associated diseases, including cancer, requires human models.

The ability to generate brain organoids derived from human pluripotent stem cells provides an unprecedented opportunity to study context-dependent human disease pathologies in an experimentally tractable system. Indeed, this approach has provided insights into alterations associated with Alzheimer's, blindness, autism spectrum disorder, Zika virus infection, and others (Lancaster \& Knoblich, 2014b; Quadrato et al, 2016; Di Lullo \& Kriegstein, 2017; Amin \& Pasca, 2018; Rossi et al, 2018; Chen et al, 2019). A variety of protocols to generate brain organoids have been developed, but the considerable variability and heterogeneity between individual organoids obtained using these methods limits the utility of the model for studying disease mechanisms or for examining the therapeutic potential of new drug candidates. Here, we establish a robust protocol to efficiently and reproducibly generate mature, consistent (i.e., uniform) human cerebral organoids (hCOs). By optimizing an established protocol for self-patterned whole-brain organoids (Lancaster et al, 2013; Lancaster \& Knoblich, 2014a), we generated phenotypically consistent forebrain organoids with reproducible morphologies and cell-type compositions. Thus, this protocol is ideally suited for studying mechanisms underlying human diseases and for investigation of potential novel therapeutic options in an experimentally tractable system.

\section{Results}

\section{Optimization of cerebral organoid production}

To establish a method to reproducibly generate uniform brain organoids (Fig 1A), we explored modifications to a previously established protocol for generating self-patterned whole-brain organoids (Lancaster et al, 2013; Lancaster \& Knoblich, 2014a), which yields organoids with variable morphology and cell type composition (Quadrato et al, 2017; Velasco et al, 2019; Yoon et al, 2019). We primarily used female $\mathrm{H} 9$ human embryonic stem cells (hESCS) and validated results in a male hESC model ( $\mathrm{H} 1$; see below). To begin, we first optimized embryoid body (EB) generation by plating singularized H9 cells into 96-well plates with variable geometries and surface coatings and quantitatively examined cell aggregates after $5 \mathrm{~d}$. In contrast to the irregular clusters observed in traditional U-bottom

\footnotetext{
${ }^{1}$ Department of Biochemistry, University of Toronto, Toronto, Canada ${ }^{2}$ Donnelly Centre, University of Toronto, Toronto, Canada ${ }^{3}$ Department of Molecular Genetics, University of Toronto, Toronto, Canada ${ }^{4}$ Center for Systems Biology, Lunenfeld-Tanenbaum Research Institute, Mount Sinai Hospital, Toronto, Canada ${ }^{5}$ Department of Pharmacology and Toxicology, University of Toronto, Toronto, Canada
}

Correspondence: liliana.attisano@utoronto.ca 
A
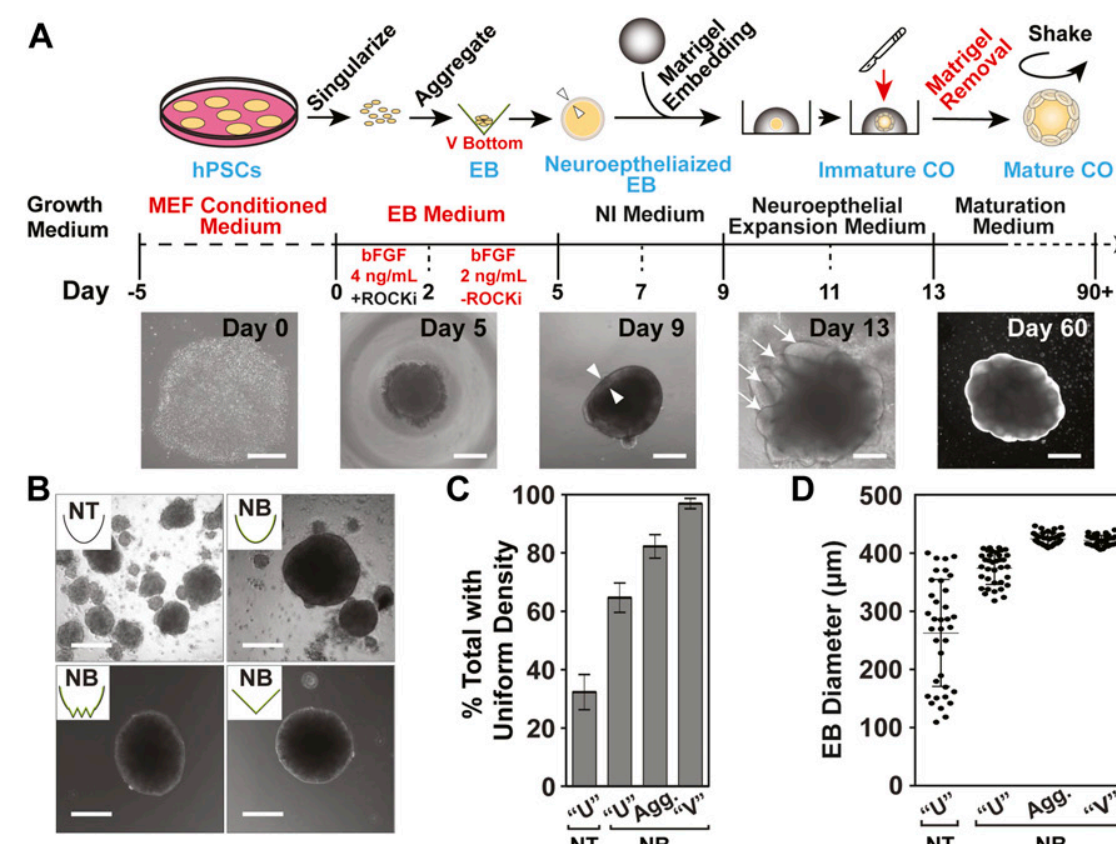

E

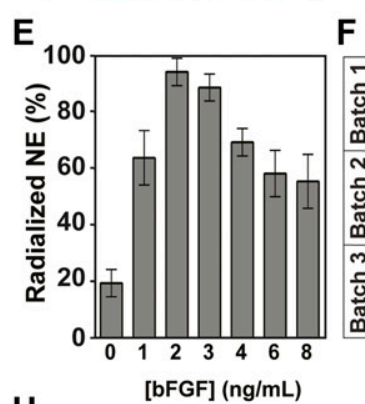

$\mathbf{F}$
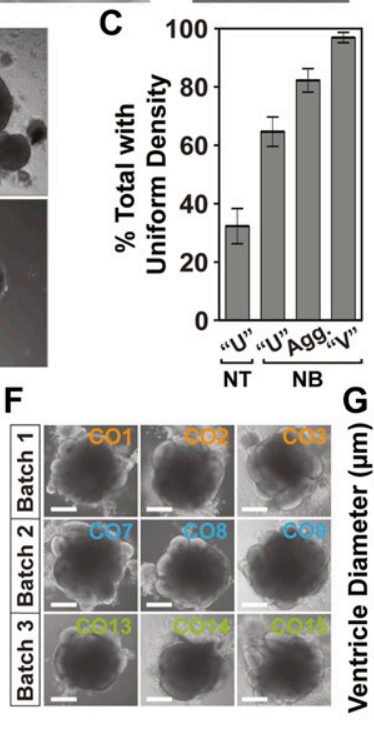

H
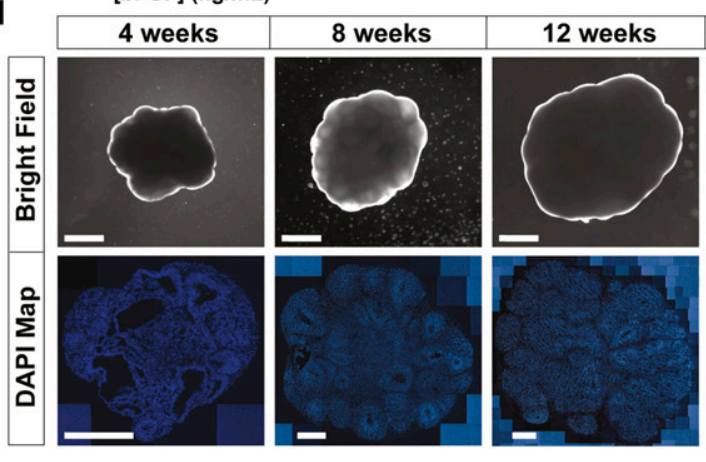

G

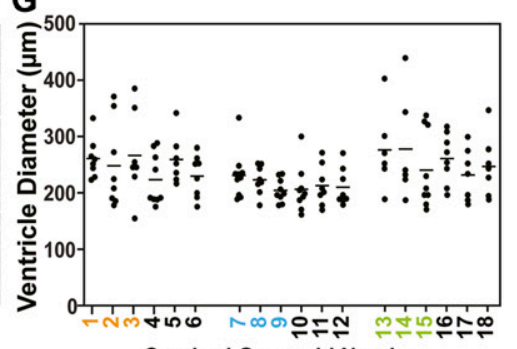

Cerebral Organoid Number

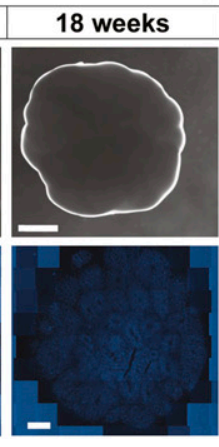

24 weeks

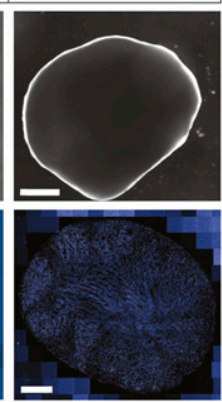

Figure 1. Generation of hCOs from H9 ESCs.

(A) A schematic depicting the main steps for human cerebral organoid (hCO) production. Representative bright-field images of morphological changes are shown below. Triangles (Day 9) mark the inner and outer edge of the neuroepithelial ring, and arrows (Day 13) indicate early ventricle structures. Scale bars: 250 $\mu \mathrm{m}$ for days $0,5,9$, and 13 and $1 \mathrm{~mm}$ for Day 60. (B, C, D) The effect of well shape and surface coating on embryoid body (EB) formation was assessed on Day 5. (B) Representative bright-field images of EBs generated using the indicated plate format. Scale bar = $250 \mu \mathrm{m}$. Non-treated (NT), nonbinding (NB). (C) Percent of cell aggregates displaying uniform density as assessed using phase-contrast microscopy is plotted as the mean $\pm S D(n=3)$. (D) Individual EB diameters (black circles) and the mean (horizontal dash) \pm SD ( $n$ $\geq 30$ /condition) is plotted. (E) Percent of total EBs displaying radialization neuroepithelium on Day 5 at the indicated bFGF concentrations are plotted as mean \pm $\mathrm{SD}(\mathrm{n}=3) .(\mathbf{F}, \mathbf{G})$ Analysis of ventricle formation on Day 13. (F) Representative bright-field images of COs before Matrigel extraction from three independent batches are shown. Scale bar $=500 \mu \mathrm{m}$. (G) Quantification of the diameter of individual ventricle-like structures (black circles) from three independent batches is plotted with the mean diameter marked (horizontal dash). (F) Colored numbering corresponds to images in panel (F). (H) Macroscopic organization in H9-derived hCOs. Representative bright-field (Scale bar $=1 \mathrm{~mm}$ ) images of hCOs in suspension culture at 4, 8, 12, 18, and 24 wk of culture (top) with corresponding sections stained with DAPI to mark nuclei (bottom). Scale bar $=500 \mu \mathrm{m}$. dishes with non-treated (unmodified polystyrene) or nonbinding (Ultra Low Attachment) surface coatings, EB aggregates that were formed in nonbinding plates with V-bottom or Aggrewell 800 (comprising multiple $V$-shaped indentations) geometries, formed similarly sized spheres of 400-450- $\mu \mathrm{m}$ diameter in each V-shaped indentation, all of which displayed similar opacity under bright-field microscopy (Fig 1B-D). Although we were able to obtain consistent EB size using both the V-bottom and Aggrewell platforms, the Aggrewell system generated multiple EBs per well which when transferred for neuralization, resulted in further aggregation of multiple EBs. For this reason, we focused on the $\mathrm{V}$-bottom nonbinding format for all subsequent studies as this streamlined selection of individual EBs.
Basic FGF (bFGF) in the presence of Nodal/Activin SMAD signalling is important for maintenance of pluripotency in hESC cells (Vallier et al, 2005), whereas in the absence of SMAD signalling, FGF drives neuroectoderm induction (LaVaute et al, 2009). However, hESC aggregates display intrinsic suppression of BMP-SMAD signalling, so the addition of FGF is sufficient to drive neural induction (Coucouvanis \& Martin, 1999; Khoo et al, 2005; LaVaute et al, 2009). Furthermore, although ROCK inhibitor (Y27) promotes survival and pluripotency of singularized hESC, it is not required for EB formation (Pettinato et al, 2014). Therefore, we removed Y27 after EBs formed ( $2 \mathrm{~d}$ ) and titrated FGF doses for the following $3 \mathrm{~d}$ to determine the optimal concentration that supports the subsequent formation of 
radialized neuroepithelium after neural induction. We observed that after $3 \mathrm{~d}$ at $2 \mathrm{ng} / \mathrm{ml}$, >90\% of EBs displayed correctly radialized neuroepithelium (i.e., a uniform, smooth clearing at the periphery: Fig S1A), which decreased to $60 \%$ at bFGF concentrations of $>6 \mathrm{ng} /$ $\mathrm{ml}$. In contrast, removal of bFGF strongly suppressed radialization to $20 \%$ (Fig $1 \mathrm{E}$ ), consistent with the important role of FGF signalling in neurectoderm induction observed in vivo, and in hESC aggregates (Vallier et al, 2005; LaVaute et al, 2009). hCOs develop as selforganizing systems, and EB size has a significant impact on differentiation trajectories, with smaller EBs favouring ectoderm (Bauwens et al, 2008; Jang \& Kim, 2010). Therefore, we also examined the effect of cell seeding density (1,000-16,000 cells/EB) on neural induction efficiency and observed peak efficiency at 12,000 cells/ well (Fig S1B). Finally, we noted that when early hCOs were extracted from Matrigel and transferred to free-floating spinning cultures, excessive Matrigel impeded the growth of morphologically uniformly shaped hCOs. This prompted us to ensure complete removal of all Matrigel from hCOs before transfer to spinning culture. For this reason, we transitioned from using the "dimpled parafilm" method (Lancaster \& Knoblich, 2014a) to embedding neuralized EBs in a Matrigel droplet immobilized on the surface of a four-well plate to facilitate complete extraction. Using all of the above optimized parameters, we assessed the morphology of 18 organoids, six from each of three separate batches on Day 13, just before transfer to spinning culture. These early organoids displayed 6-10 outer ventricle-like ring structures with an average diameter of approximately $250 \mu \mathrm{m}$ in all batches (Fig $1 \mathrm{~F}$ and G). We also noted a marked absence of the fluid-filled cyst structures (Fig S1C) commonly seen with other protocols (Lancaster et al, 2013; Lancaster \& Knoblich, 2014a). Thus, by optimizing early EB formation and the neural induction phases of organoid production, we reproducibly generated morphologically uniform hCOs with a cumulative efficiency of $80-90 \%$, while restricting unwanted non-neural differentiation (Fig S1D). Similar results were also obtained using H1 ESCS (Fig S1E-G).

\section{Characterization of cerebral organoid development and maturation}

On Day 14, early organoids coated in Matrigel had a diameter of approximately $500 \mu \mathrm{m}$, and after careful excision from the Matrigel, they were transferred to spinning culture where they were left to mature as free-floating structures. By 12 wk, hCO diameters increased to roughly $3 \mathrm{~mm}$ after which little additional growth was observed for up to $24 \mathrm{wk}$ of culture (Fig $1 \mathrm{H})$. With the exception of an occasional loss due to the fusion of two organoids, virtually all hCOs continued to maturity. To assess morphogenesis, we next evaluated cell type-specific marker gene expression by immunofluorescence microscopy. Distinct ventricle-like structures (ventricular units) were abundant in early-stage hCOs (4-12 wk) and diminished in later stages (18 and 24 wk). Serial sectioning of ventricular units showed them to be spherical with a hollow center reminiscent of the apical space in ventricles in the developing cortex (Fig S2A). Staining with SOX2, which marks radial glial (RG) cells, showed expression in cells lining the ventricular space similar to in vivo (Figs 2A and S2B), with most ventricular units displaying robust SOX2+ staining at 4 wk. However, by 12 wk, SOX2+ ventricular units

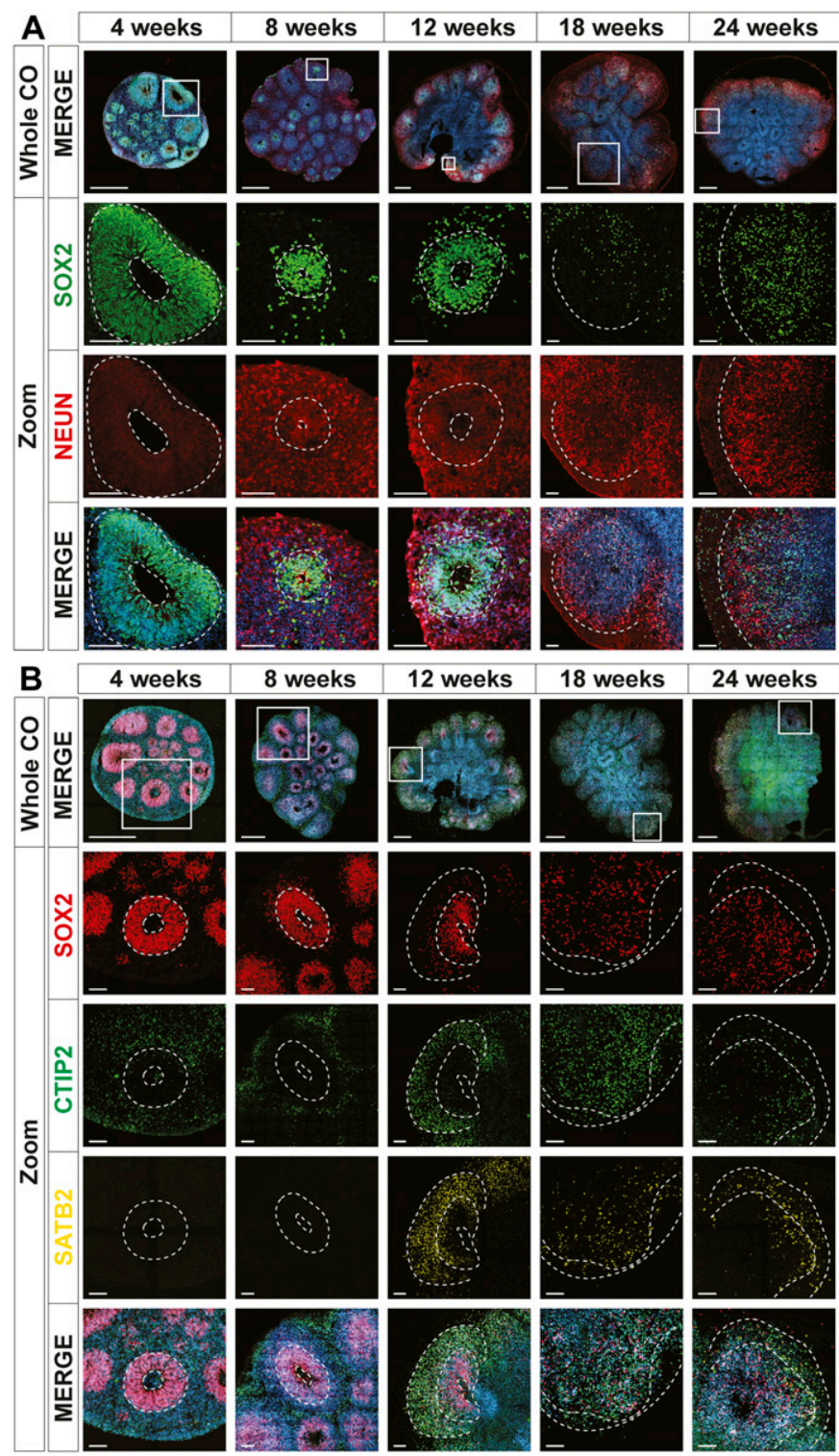

Figure 2. Human cerebral organoids (hCOs) mimic early human cortical development.

The localization of SOX2 (radial glia), NeuN (neurons), CTIP2, and SATB2 (cortical layer markers) in hCOs at 4, 8, 12, 18, and 24 wk of age, co-stained with DAPI, was visualized by immunofluorescence microscopy. Images of whole hcOs (top) and magnified images (Zoom) of selected ventricles or regions (white box) at each time point are shown. White dashed lines mark ventricles (center ring) and the outer perimeter SOX+ radial glial cells (outer ring). White dashed lines mark the ventricle-like cavities (inner ring) and the outer perimeter of the SOX2+ layer in 4-12-wk COs or the SOX+ and outer edge of the cortical plate in 18- and 24-wk COs. (A) Ventricle-like structures are lost in older (18 and 24 wk) COs. (B) Expression of CTIP2 (gene name, BCL11B; deep layer cortical neuron marker) precedes that of SATB2 (upper layer cortical neuron marker), both of which are superficial to the SOX2+ ventricular zone in 4-12-wk COs, recapitulating in vivo cortical development, whereas in older COs (18 and $24 \mathrm{wk}$ ), this distinct separation is less evident. Scale bar $=500 \mu \mathrm{m}$ for whole COs and $100 \mu \mathrm{m}$ for magnified ventricles.

were more variable and in older hCOs were localized to the outer regions. In contrast, NeuN, which marks mature neurons, was low in 4-wk-old hCOs but was readily detected in hCOs 8 wk or older, 
where it was present outside of the SOX2 expression domain (Figs $2 \mathrm{~A}$ and $\mathrm{S} 2 \mathrm{~B}$ ). Similar to the in vivo orientation, TUJ1, a neurofilament protein, marked RG processes running perpendicular to the ventricular zone as early as 4 wk (Fig S3A). Outside of the RG cells, parallel TUJ1+ processes were observed in superficially localized neurons. The cortical layer markers, CTIP2 (gene name, BCL11B) and SATB2, which identify layers V (deep) and II-IV (upper), respectively, were also detected superficial to SOX2 staining in early hCOs, with CTIP2 detected as early as $4 \mathrm{wk}$, and SATB2 first evident at $12 \mathrm{wk}$ (Figs $2 \mathrm{~B}$ and $\mathrm{S} 3 \mathrm{~B}$ ). This temporal sequence is consistent with the timing of in vivo development in which formation of deep layers precedes that of upper layer neurons. These studies further revealed that in older hCOs, although markers of RG cells and mature neurons were maintained, the cells expressing the markers became intermingled, and the organized layering of ventricular units evident in earlier hCOs was lost, as reported previously (Watanabe et al, 2017). Altogether, this analysis demonstrates that the hCos generated using the optimized pipeline display organization and patterning reminiscent of that observed in vivo (Molnar et al, 2019).

\section{Single-cell profiling of hCOs}

To gain a comprehensive view of cell types present in individual hCOs, we performed single-cell RNA sequencing (scRNA-seq) on individual organoids at 12,18 , and 24 wk of culture. Unsupervised clustering was performed on the gene expression profiles and data were visualized using Uniform Manifold Approximation and Projection (UMAP) plots, with single-cell metrics and sequencing data quality summarized in Table S1. Cell types were then identified by comparing the differentially expressed genes in each of the clusters to known cell type-specific marker genes. Profiling of six organoids derived from three separate batches at 12 wk of age identified 15 distinct clusters (Figs $3 \mathrm{~A}-\mathrm{C}$ and $\mathrm{S4}$ ). This included RG cells (SOX2, PAX6, HES1, and GLI3) of both proliferative (proRG: cluster 13, MKI67, CENPF, and TOP2A) and outer RG (oRG; cluster 9, HOPX, FAM107A, TNC, and LIFR) subtypes (Pollen et al, 2015), with some overlap between these classes, consistent with RG cell function. RG cells give rise to neurons by transitioning through a transcriptionally distinct intermediate progenitor cell state marked by the expression
A

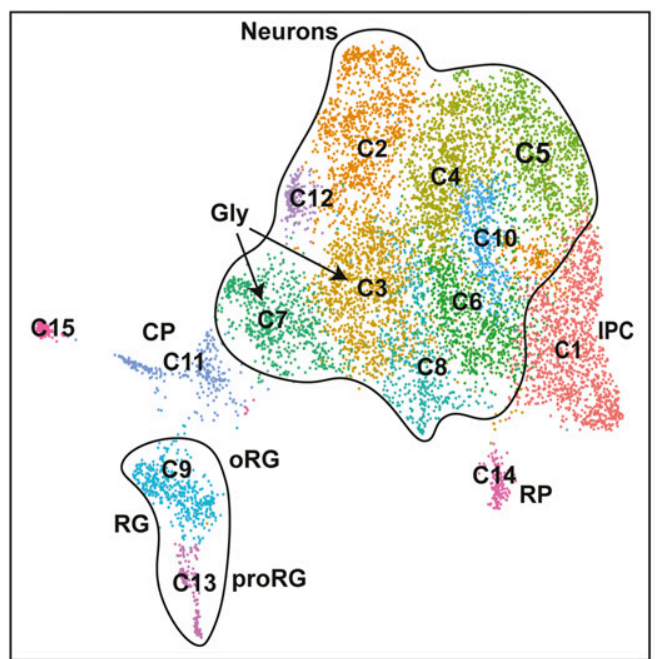

C
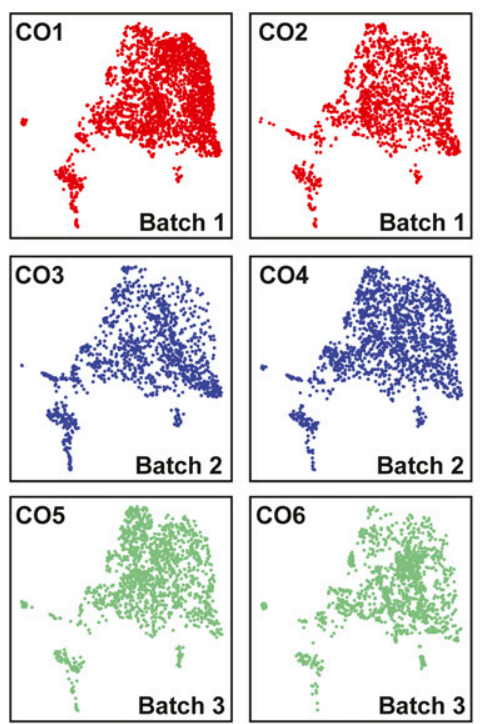

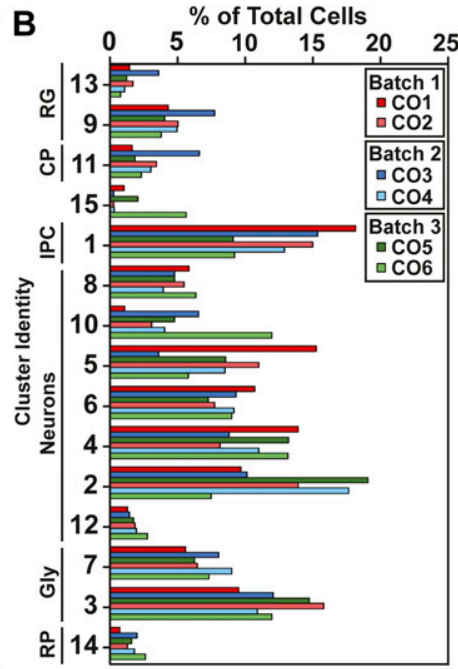

D

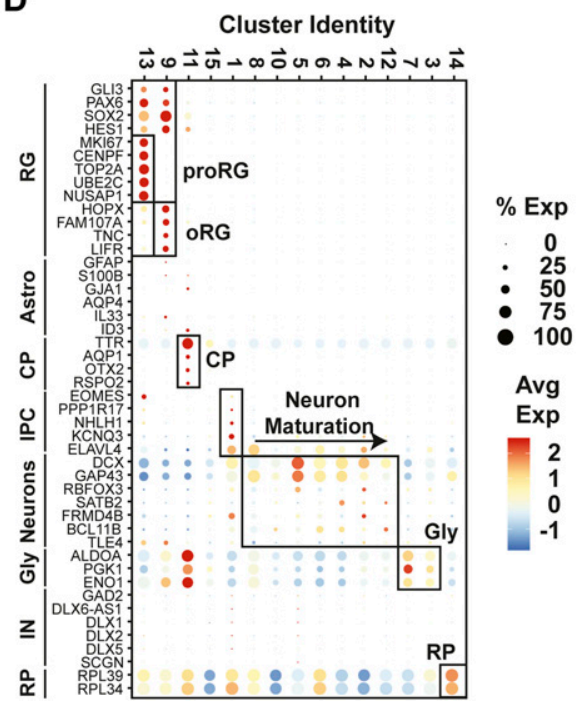

Figure 3. Uniform cell type composition in 12 human cerebral organoids ( $\mathrm{hCOs}$ ) is revealed by single-cell RNA sequencing.

(A) A Uniform Manifold Approximation and Projection plot from unsupervised clustering of single-cell RNA sequencing data from six 12-wk-old hCOs obtained from three separate batches (CO1-CO6) (10,985 cells) is shown. Cluster identities are indicated. (B) Cluster frequency analysis depicting the percentage of cells in each individual organoid that contributed to each cluster. (A, C) Individual 12-wk-old hCOs (CO1-CO6) are plotted in the Uniform Manifold Approximation and Projection axis defined in panel (A). (A, D) A dot blot indicates the expression of cell type-specific marker genes for all clusters in panel (A). 
of EOMES and PPP1R17 (Pollen et al, 2015), which mapped to cluster 1. Neurons comprised the majority (74\%) of the cells, encompassing clusters 2-8, 10, and 12. These included immature neurons expressing high levels of DCX and GAP43, as well as more mature neurons that expressed deep and superficial layer markers such as SATB2 and $B C L 11 B$ (CTIP2). Two of the neuronal clusters ( 3 and 7) displayed a particularly high level of glycolytic (Gly) marker genes (ALDOA, PGK1, and ENO1), a neuronal subset also recently reported in hCOs (Pollen et al, 2019). Interestingly, cells expressing markers of mature astrocytes (GFAP and S100B) were rare (Fig 3D), suggesting neurogenesis is the primary process occurring in the first $12 \mathrm{wk}$ of culture. In addition to RG cells and neurons, we identified cluster 11 as choroid plexus (CP), with cells expressing characteristic CP markers (TTR, AQP1, OTX2, and RSPO2), whereas in one small cluster (\#14), the differentially expressed genes were almost exclusively ribosomal proteins. Of note, the hCOs comprised predominantly dorsal forebrain neurons as markers of midbrain and hindbrain (HOXA3, HOXB3, IRX2, EN2, PAX2, and GBX2) were not detected. Moreover, there was a complete absence of markers identifying mesenchymal lineages (MYOG, MYH1, DCN, and BGN) or retinal cells (OPN1SW, RCVRN, TULP1, and $R O M 1$ ) indicating the fidelity of our optimized pipeline in promoting forebrain-specific differentiation.

We next compared cell type composition in individual organoids to assess inter-organoid variability. For this, the percent of cells in each organoid that contributed to each of the 15 clusters was determined. This analysis revealed a remarkable conservation of cell type composition amongst the individual organoids (cluster frequency variation of $<5 \%$ ) even when derived from independent batches (Fig 3B and C). Importantly, all COs contained cells from all of the clusters, indicative of conserved developmental trajectories. Transcriptional profiling of older hCOs of 18 wk (three hCOs from three batches) and 24 wk (eight hCOs from three batches) was also performed (Fig S5-S8). Like 12-wk-old hCOs, cell type composition was similar between individual hCOs across multiple organoids and batches at both 18 and 24 wk, with a median cluster frequency variation of $3 \%$ and $15 \%$, respectively (Figs S5A-D and S7A-D). Thus, even upon prolonged culturing, our optimized pipeline yields organoids of similar composition and morphology.

\section{Analysis of cell type diversity in maturing hCOs}

To track cell composition in maturing organoids, the transcriptional profiles of 12-, 18-, and 24-wk-old organoids were combined and plotted in a single UMAP comprising 19 clusters (Figs 4A, S9A and B, and S10). Analysis of the relative contribution of cells from each time point (Figs 4B and C and S9A) revealed the emergence at $24 \mathrm{wK}$ of a new cluster comprised interneurons (IN; expressing DLX1, DLX2, $D L X 6, D L X 6-A S, G A D 1$, and GAD2). Of note, these interneurons lacked expression of ISL1 and EBF1, suggesting caudal/medial identity, rather than lateral ganglionic eminence (Wamsley \& Fishell, 2017). These INs cluster close to the intermediate progenitor cell population in the UMAP, suggesting they may have arisen from these progenitors (Fig 3D). We also noted another emerging subpopulation of cells within the RG/astroglia cluster (cluster 6; circle) in
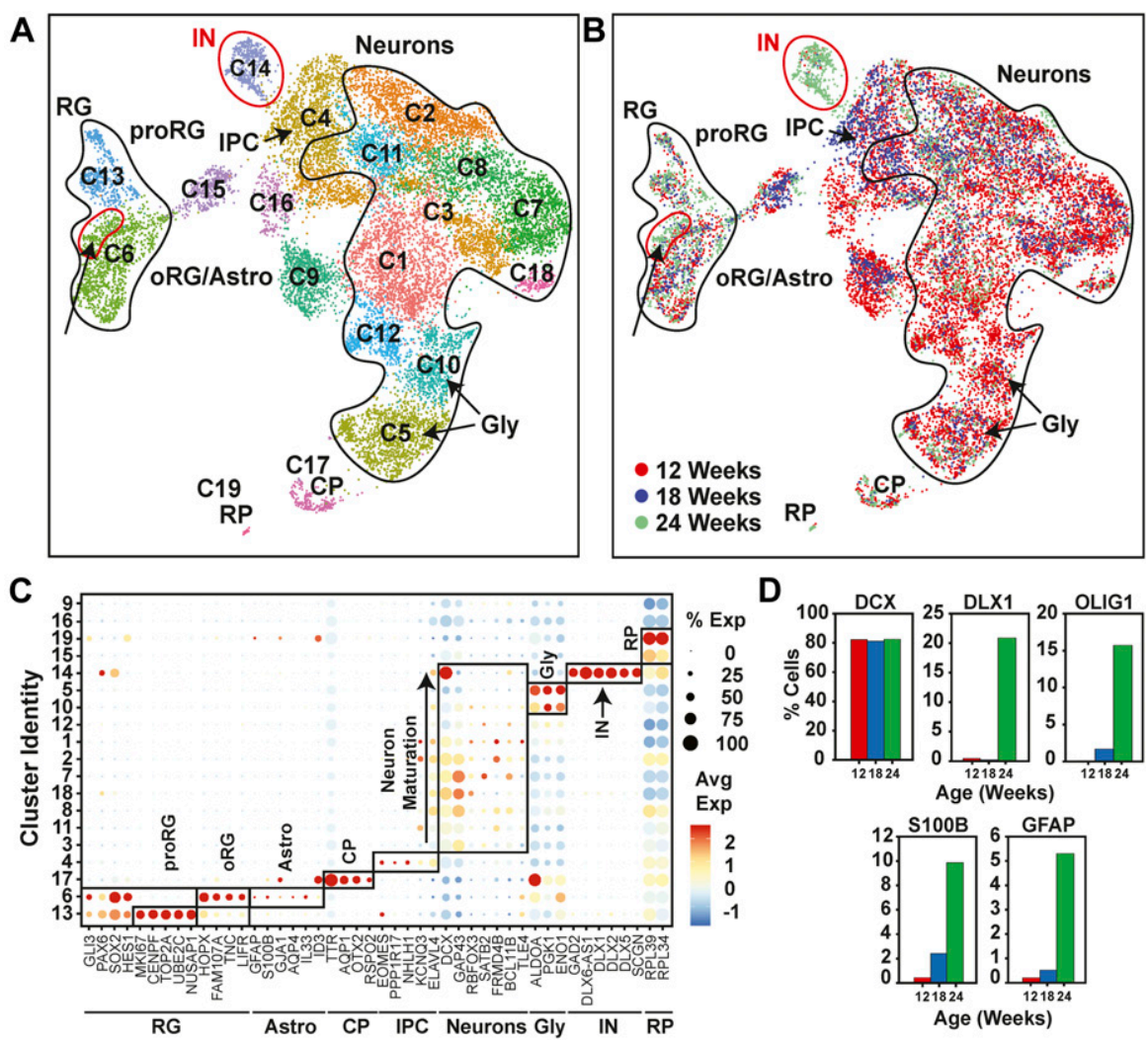

Figure 4. Characterization of cell type maturation in human cerebral organoids using single-cell RNA sequencing.

(A) Unsupervised clustering of combined single-cell RNA sequencing data from 12-, 18-, and 24-wk-old COs was visualized in Uniform Manifold Approximation and Projection plots. Cell types appearing at 24 wk, including interneurons (IN) in cluster 14 (red circle) and oligodendrocyte progenitors in oRG/astroglial cluster 6, (arrow and red circle) are marked. Cluster identities are indicated. (A, B) Uniform Manifold Approximation and Projection present in (A) segregated by time point. Cells from 12 wk depicted in red, blue for 18 wk, and green for 24 wk. (A, C) A dot blot indicates the expression of cell type-specific marker genes for all clusters in panel (A). The percent of cells expressing the gene (circle diameter) and the scaled average expression of the gene is indicated by the colour. (D) The percent of cells with expression of the indicated cell lineage markers, including $D C X$ (neurons), S100B (mature astrocytes), GFAP (astroglia/ astrocytes), DLX1 (interneurons), and OLIG1 (oligodendrocyte precursors) and at 12, 18, and 24 wk time points is plotted. Note the general neuronal marker $D C X$, which is expressed similarly across the time points, was used as a reference. $\mathrm{CP}$, choroid plexus; GLY, glycolytic signature; IN, interneurons; IPC, intermediate progenitor cells; ORG, outer radial glial cells; proRG, proliferative radial glial; RG, radial glial cells; RP, ribosomal protein. 
24-wk-old hCOs (Fig 4 A and B). This population was marked by OLIG1 and OLIG2 expression, suggesting an oligodendrocyte precursor population (Figs 4A-D and S9B). RG cells are known to produce both neurons and glia, although few cells $(<1 \%)$ in the 12 -wk-old hCOs expressed markers of mature astrocytes (GFAP and S100B; Figs 4D and $\mathrm{S} 9 \mathrm{~B}$ and $\mathrm{C}$ ). However, cells with an astroglial identity (Astro) were detected within the oRG cluster starting at 18 wk (Fig S5, cluster 5) that further increased by 24 wk (Fig S7A, cluster 3), indicating the emergence of mature astroglia from RG cells (Figs 4D and $\mathrm{S} 9 \mathrm{~B}$ and C). Overall, this suggests that the rate of gliogenesis increases upon longer culturing, which parallels the temporal regulation of cortical development in vivo (Sauvageot \& Stiles, 2002; Pinto \& Gotz, 2007).

\section{Electrophysiological analysis}

To assess neuronal function, electrophysiological output was measured by whole cell patch clamping of individual neurons in fresh slices prepared from hCOs at 12 and 24 wk. Patched neurons displayed varying degrees of neurite networks as visualized by biocytin labelling (Fig 5A). Analysis of electrical properties identified three types of neurons, namely, immature, developing, and mature. Immature neurons did not fire action potentials (AP; data not shown), developing neurons, fired APs but with slow kinetics and small amplitudes, and mature neurons, fired APs with fast kinetics and high amplitudes and generated stable trains of spontaneous APs (Fig 5B and C). Both developing and mature neurons also
A

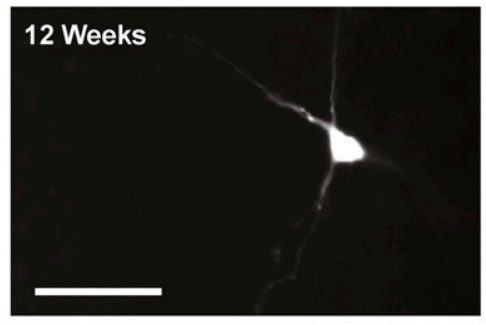

B

C

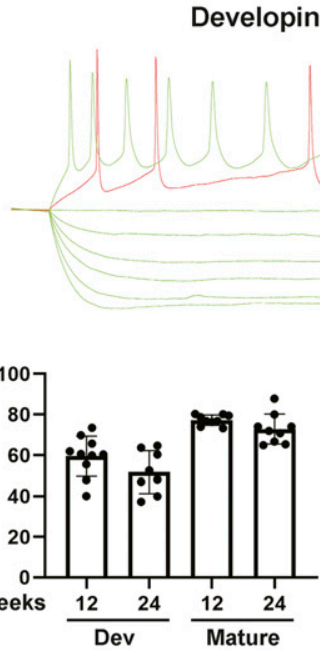

D

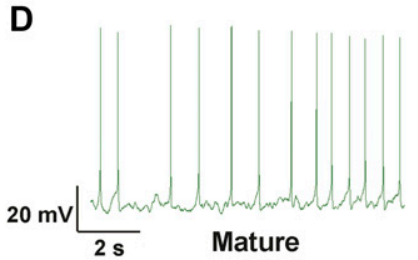

$\mathbf{F}$

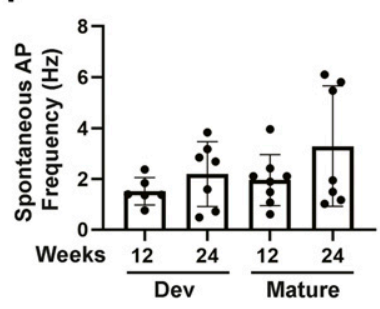

$20 \mathrm{mV}$ $100 \mathrm{~ms}$

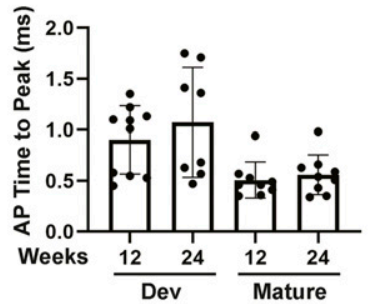

E
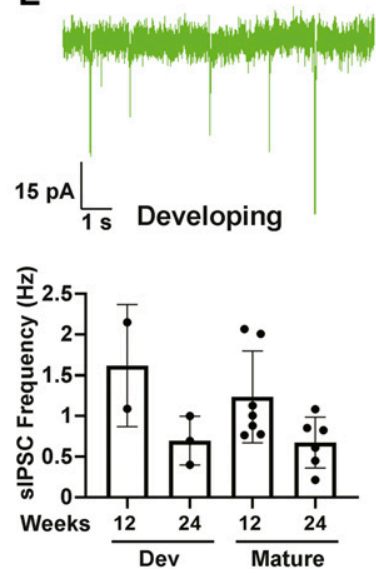
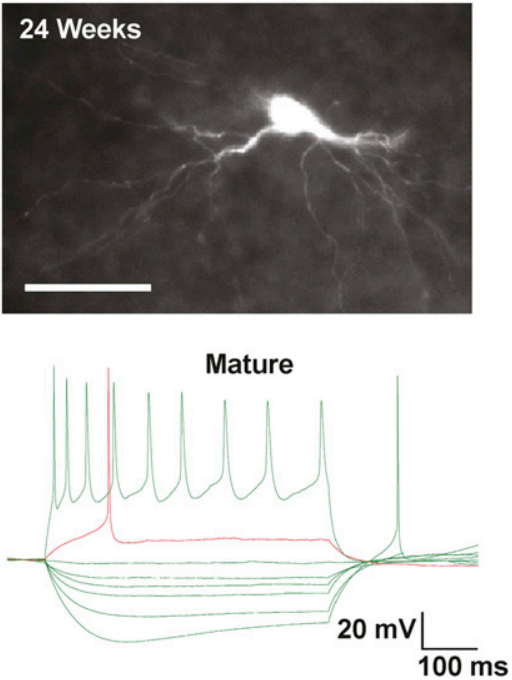

$100 \mathrm{~ms}$
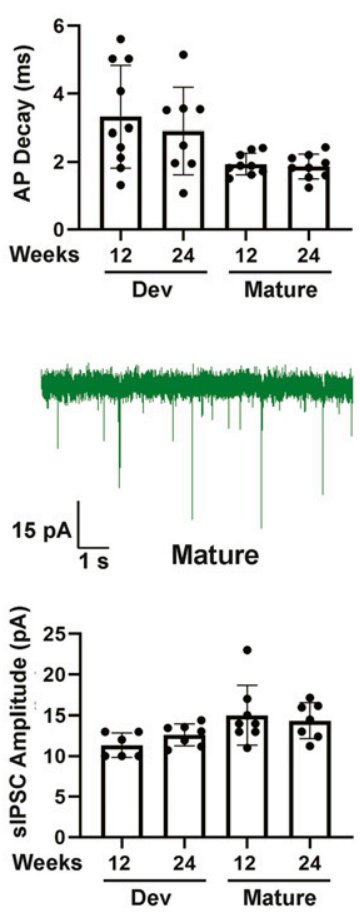

Figure 5. Electrophysiological analysis of 12- and 24wk-old human cerebral organoids using whole cell patch clamping.

(A) Images of recorded developing or mature neurons marked with biocytin taken using 40x water immersion objective are shown. Scale bar $=20 \mu \mathrm{m}$. (B) Representative traces from whole cell patch clamping of developing and mature neurons are shown. (C) Peak action potential amplitude \pm SD, mean time to peak action potential amplitude \pm SEM, and mean action potential decay time \pm SEM, for individual recordings are plotted for 8-10 neurons per condition. (D, E) Characterization of spontaneous currents. (D, E) A representative trace of spontaneous AP firing in a mature neuron (D) and of mean frequency \pm SEM of spontaneous excitatory postsynaptic currents in developing and mature neurons (E) are shown. (F) Frequency and amplitude of spontaneous IPSCs in developing and mature neurons are plotted as the mean \pm SEM for 2-6 neurons per condition. 
displayed sodium and potassium currents, although they were smaller in the developing neurons (Fig S11). Moreover, only mature neurons generated stable spontaneous APs upon slight depolarization although both developing and mature neurons displayed similar spontaneous AP frequency and amplitude (Fig 5D-F). Of note, all three neuron classes were present in both 12- and 24-wk old hCOs, indicating that even at 12 wk, electrophysiologically mature neurons were present.

\section{Discussion}

Human stem cell-derived cerebral organoids provide an unparalleled model system to study human neocortical development and associated disease processes. However, a key limitation for research applications is the considerable variability in shape/ architecture and cell type composition present in individual organoids. This characteristic makes it particularly challenging to design experiments to address the effects of genetic variants, therapeutic candidates, and other perturbations on CO morphogenesis or function that allow statistically supported conclusions to be drawn. Here, we describe a protocol to efficiently generate human dorsal forebrain organoids with phenotypically uniform morphologies that are reproducibly comprised of similar proportions of cell types across independent batches. This highly reproducible model system is, thus, amenable for the study of pathways controlling early human brain development and disease processes.

Several protocols have been developed to generate cerebral organoids, including both directed and self-directed. For example, several groups use modified versions of a directed approach established by the Sasai group, sometimes referred to as cortical spheroids, in which small molecules that modulate the SMAD and Wnt pathways and the growth factors EGF and FGF2 are used to achieve directed differentiation (Kadoshima et al, 2013; Velasco et al, 2019; Yoon et al, 2019). Here, we selected a previously established method of self-patterned organoids (Lancaster et al, 2013; Lancaster \& Knoblich, 2014a) as a starting point. Our work to standardize production revealed that optimization of the early steps of organoid generation, including the EB formation and neural induction phases, was key in ensuring the production of similar COs. This included normalizing EB morphogenesis using early removal of ROCKi and optimized FGF treatment, cell numbers, and well geometry and coatings. Finally, we found that efficient removal of Matrigel before spinning culture improved subsequent development. Applying this method, we demonstrated using single-cell profiling that our individual cOs have extensive cell diversity but with similar proportions of cell types even across different batches. In contrast, the reported single-cell analysis of the originally established method revealed considerable variability in the proportions of cell types present in individual organoids, particularly across different batches (Quadrato et al, 2017). Furthermore, we observed uniform distribution of ventricular units, and that the cell type composition evident in 12wk-old hCOs was maintained in older organoids, with additional cell types, including interneurons, mature astrocytes, and oligodendrocyte precursors emerging at 24 wk. Importantly, these new cell types were present in all organoids at similar proportions. Finally, our electrophysiological analysis revealed the presence of mature, electrically active neurons.

Our optimized self-directed protocol that generates highly reproducible hCOs complements those reported in other recent studies that use a directed patterning approach involving small molecule inhibitors and growth factors (Kadoshima et al, 2013; Velasco et al, 2019; Yoon et al, 2019). The availability of several robust platforms to produce highly similar hCOs, including the one described herein, thus allows for systematic molecular and cellular characterization of the development of the human cortex and how disease-causing mutations alter development and perhaps also homeostatic events.

\section{Materials and Methods}

\section{Cell line generation and culturing}

$\mathrm{H} 9$ and $\mathrm{H} 1 \mathrm{ESCS}$ were cultured on plates coated with hESC-grade Matrigel (\#CA89050-192; VWR) at $37^{\circ} \mathrm{C}$ and $5 \% \mathrm{CO}_{2}$ in mitotically arrested MEF-conditioned media, DMEM/F12 (\#11330057; Life Technologies), 20\% KnockOut Serum Replacement (KSR, \#A3181502-02; Life Technologies), 2\% MEM-NEAA, $55 \mu$ M $\beta$-mercaptoethanol (\#21985023; Life Technologies), and $4 \mathrm{ng} / \mathrm{ml}$ basic FGF (bFGF, \#100-18B; Peprotech). The medium was changed daily and the cells were split at a 1:6 ratio using Collagenase IV (\#07909; Stemcell Technologies) every 5-6 d.

\section{Generation of cerebral organoids}

ESCs were singularized using TrypLe Select (\#12563011; Life Technologies) and resuspended at 80,000 cells/ml in EB media (DMEM/ F12, 20\% KSR, 2\% MEM-NEAA, $55 \mu \mathrm{M} \beta$-mercaptoethanol) with $4 \mathrm{ng} /$ $\mathrm{ml} \mathrm{bFGF} \mathrm{and} 50 \mu \mathrm{M}$ Y-27632 (Y27) (\#S1049; Selleck Chem). The cells (12,000 cells/well) were plated in 96-well V-bottom nonbinding plates (\#651970; Greiner Bio-One), and on Day 2, fresh EB medium containing $2 \mathrm{ng} / \mathrm{ml}$ bFGF was added. On Day 5, healthy EBs with a diameter of 425-475 $\mu \mathrm{m}$ were transferred to 24-well ultralow attachment plates (\#CLS3473; Corning) in $500 \mu \mathrm{l}$ neural induction medium (DMEM/F12, 1\% N2, 1\% MEM-NEAA, 1\% GlutaMax, and $1 \mu \mathrm{g} /$ $\mathrm{ml}$ heparin [\#H3393; Sigma-Aldrich]), and $48 \mathrm{~h}$ later, an additional $500 \mu \mathrm{l}$ of neural induction medium was added. EBs were transferred to pre-warmed four-well tissue culture plates, excess medium was aspirated, and freshly thawed growth factor reduced Matrigel (30 $\mu \mathrm{l}$ ) was added on top of EBs. Plates were transferred to a $37^{\circ} \mathrm{C} \mathrm{CO}_{2}$ incubator for $10 \mathrm{~min}$ to allow the Matrigel to polymerize, and then $500 \mu \mathrm{l}$ of cerebral organoid differentiation media without vitamin A (CDM - Vit A; 48\% DMEM/F12, 48\% neurobasal [\#21103049; Life Technologies], 0.5\% MEM-NEAA, 1\% GlutaMax, 0.5\% N2, 1\% B27 without vitamin A [\#12587001; Life Technologies], $2.5 \mu \mathrm{M}$ insulin, and $192.5 \mu \mathrm{M} \beta$-mercaptoethanol) was added to each well. On Day 11, the medium was replaced, and on Day 13 , spheroids containing ring-like structures were extracted using a Scalpel (\#1000044; Thermo Fisher Scientific), ensuring that excess Matrigel is removed. Organoids (maximum of three per well) were transferred to a six-well tissue 
culture plate containing $3 \mathrm{ml}$ of CDM + Vit A (CDM with 1\% B27 containing Vit A) and then placed on an orbital shaker ( $\# 88881101$; Thermo Fisher Scientific) at $90 \mathrm{rpm}$ (9.5 mm radius) in a $\mathrm{CO}_{2}$ incubator for the duration of culturing. The medium $(2 \mathrm{ml})$ was replaced every $72 \mathrm{~h}$ for the first $30 \mathrm{~d}$ and then increased to $3 \mathrm{ml}$ for the duration of culture. The plates were replaced every $30 \mathrm{~d}$ to prevent buildup of debris. Spheroid formation efficiency was tested using nonbinding V-bottom plates (\#651970; Greiner Bio-One), Aggrewell800 plates (\#38421; StemCell Tech) coated with AntiAdherence Rinsing Solution (\#07010; StemCell Tech), U-bottom non-treated polystyrene plates (\#168136; Thermo Fisher Scientific), and U-bottom Ultra-Low Attachment plates (\#4515; Corning).

\section{Immunofluorescence microscopy}

COs were washed three times for 5 min in $5 \mathrm{ml}$ of PBS in 5-ml roundbottom tubes (\#1152367; BD Falcon) and then fixed overnight in $4 \%$ PFA at $4^{\circ} \mathrm{C}$ on a rocker. The samples were washed three times for 5 min in $0.1 \%$ Tween20 in PBS (PBST) and then sequentially soaked in $15 \%$ sucrose solution for $2 \mathrm{~h}$ and then $30 \%$ sucrose solution overnight $\left(4^{\circ} \mathrm{C}\right)$. Organoids were transferred to a cryomold containing OCT (\#95057-838; VWR) and flash-frozen in liquid nitrogen using a stainless steel bucket containing 2-methylbutane (\#M32631; Sigma-Aldrich). The samples were sectioned to $20 \mu \mathrm{m}$ using a Leica CM3050S cryostat, mounted on SuperFrost Plus Microscope Slides (\#22-037-246; Thermo Fisher Scientific), and heated for $15 \mathrm{~min}$ at $45^{\circ} \mathrm{C}$. The samples were washed three times in PBST at room temperature, blocked, and permeabilized for $1 \mathrm{~h}$ at room temperature in 2\% BSA and $0.5 \%$ Triton-X100 diluted in PBST (PBSTX) in a humidified chamber. Primary antibodies listed in Table S2 were diluted in cold PBST containing $0.5 \%$ BSA and incubated overnight at $4^{\circ} \mathrm{C}$, washed three times for $5 \mathrm{~min}$ in PBST at room temperature, and then incubated in Invitrogen DyLight Secondary Antibodies (1: $500)$ and DAPI $(1: 2,000)$ in PBST containing 0.5\% BSA overnight at $4^{\circ} \mathrm{C}$. The slides were washed in PBST three times for $10 \mathrm{~min}$ and mounted using MOWIOL-DABCO Mounting Media (\#10891; SigmaAldrich) and then dried at room temperature overnight in a dark box. The samples were imaged using a Nikon T2i microscope with a Hamamatsu confocal camera or Zeiss CSU-X1 confocal microscope. Images were processed using NIS elements (Nikon Elements) software or Volocity (PerkinElmer) software, respectively.

\section{Paraffin embedding}

COs were washed two times for $5 \mathrm{~min}$ at room temperature in $5 \mathrm{ml}$ of PBS in 5-ml round-bottom tubes (\#1152367; BD Falcon) and then fixed overnight in $4 \%$ PFA at $4^{\circ} \mathrm{C}$ on a rocker. The samples were washed three times for $15 \mathrm{~min}$ in PBS and then sequentially dehydrated in $40 \%, 70 \%$, and $95 \%$ ethanol diluted in $\mathrm{ddH}_{2} \mathrm{O}$ for $1 \mathrm{~h}$ each, $95 \%$ ethanol overnight, and then three times for $1 \mathrm{~h}$ in $100 \%$ ethanol at $4^{\circ} \mathrm{C}$ on a rocker. COs were then soaked in xylene for 5-10 min at room temperature in biopsy cassettes (\#M506-3; Simport). The samples were air-dried for 2-3 min and washed twice in melted paraffin ( \#39602004; Leica) at $60^{\circ} \mathrm{C}$ for $1 \mathrm{~h}$ and once overnight. The samples were embedded into paraffin blocks within $16 \mathrm{~h}$, sectioned using 80-mm microtome blades (MB35; Thermo Fisher Scientific) at 14 or $20 \mu \mathrm{m}$ on a semiautomated rotary microtome (RM2245; Leica), mounted on SuperFrost Plus Microscope Slides in distilled $\mathrm{H}_{2} \mathrm{O}$ heated to $45^{\circ} \mathrm{C}$, and then dried overnight at $45^{\circ} \mathrm{C}$ and stored at $4^{\circ} \mathrm{C}$.

\section{Hematoxylin and eosin staining}

Paraffin slides were deparaffinized by two washes of xylene, $100 \%$, $100 \%, 95 \%$, and $70 \%$ ethanol for 5 min each. Deparaffinized or cryopreserved slides were soaked for 5 min in $\mathrm{dH}_{2} \mathrm{O}$, stained with Harris' Hematoxylin (\#HHS32; Sigma-Aldrich) for 3 min, rinsed in warm running tap water for $4 \mathrm{~min}$, and then de-stained in $0.3 \%$ acid ethanol $(70 \%$ ethanol and $0.3 \% \mathrm{HCl})$ for $30 \mathrm{~s}$ and rinsed in warm running tap water for $5 \mathrm{~min}$. The samples were dipped in the bluing agent $0.1 \%$ sodium carbonate (\#1613757; Sigma-Aldrich), washed in running tap water for $2 \mathrm{~min}$, and then counterstained in a working solution of $0.25 \%$ eosin (in $80 \%$ ethanol and $0.5 \%$ glacial acetic acid) for $20 \mathrm{~s}$. The slides were sequentially dehydrated for $1 \mathrm{~min}$ in $70 \%$, $95 \%$, and $100 \%$ ethanol and for 5 min in xylene or Histo-Clear II (\#HS202; National Diagnostics), mounted using Histomount (\#008030; Thermo Fisher Scientific), and left to dry overnight before imaging. H\&E images were taken on a dissection scope with a mounted camera or with a 20× objective on Axio Scan.Z1 (Zeiss) and processed using Zen (Zeiss) software.

\section{Sample preparation for scRNA-seq}

COs were washed three times for 1 min each in with $5 \mathrm{ml}$ of roomtemperature PBS, cut into fragments, digested with Accutase at $37^{\circ} \mathrm{C}$ for $20 \mathrm{~min}$, with pipetting every $10 \mathrm{~min}$ to facilitate cell dissociation. The cell suspension was passed through a 40-micron filter, which was then washed once with $2 \mathrm{ml}$ of fresh Accutase to collect remaining single cells and then centrifuged at $120 \mathrm{rcf}$. The pellet was resuspended in $5 \mathrm{ml}$ of Accutase at room temperature. Live cells were counted using trypan blue staining, centrifuged at 120 rcf for $5 \mathrm{~min}$, and single cells were resuspended in CDM + Vit A at 500,000-1,000,000 cells/ $\mathrm{ml}$ and trypan blue-positive cells recounted. The samples were placed on ice and then subjected to barcoding using the 10x Chromium platform following the manufacturer's recommendations. cDNA libraries were sequenced on either Illumina's HiSeq 3000 or a NovaSeq 6000.

\section{Analysis of scRNA-seq data}

SCRNA-seq were processed with Cell Ranger (V2.1) software (10× Genomics) to perform demultiplexing, unique molecular identifier collapsing, demultiplexing, and alignment to the GRCh38 human transcriptome. Post-processing of the raw Cell Ranger output matrix was performed using an in-house pipeline (Ayyaz et al, 2019). Data processing including cell filtering using a stringent filter, gene filtering, and data normalization for individual samples were performed with scater (McCarthy et al, 2017). Merging of multiple samples into a combined object, unsupervised clustering, visualizations, and differential expression analysis was performed using Seurat (Butler et al, 2018; Stuart et al, 2019). In Seurat, UMAP was used as the preferred dimensionality reduction method (Becht et al, 2018) whereas MAST was used for differential expression analysis (Finak et al, 2015). Batch correction between multiple batches in the same time point as well as between time points was performed 
using Harmony (Korsunsky et al, 2018 Preprint). All data plotting and analyses were performed in R (www.R-project.org).

\section{Electrophysiological recordings}

COs $(500 \mu \mathrm{m})$ were embedded in $0.4 \%$ low-melting agarose (\#16520100; Thermo Fisher Scientific) diluted in PBS and then sectioned using a Leica VT1200S vibratome. The slices were perfused at a constant rate of $2 \mathrm{ml} / \mathrm{min}$ with oxygenated, warmed recording artificial cerebrospinal fluid solution, containing $124 \mathrm{mM}$ $\mathrm{NaCl}, 25 \mathrm{mM} \mathrm{NaHCO}_{3}, 2.5 \mathrm{mM} \mathrm{KCl}, 2.5 \mathrm{mM} \mathrm{MgCl}$, $1.2 \mathrm{mM} \mathrm{CaCl}_{2}$, and 10 $\mathrm{mM}$ glucose at a temperature $32^{\circ} \mathrm{C} \pm 1^{\circ} \mathrm{C}$. Visually guided whole-cell patch-clamp recordings were obtained from neurons with a patch solution containing $120 \mathrm{mM} \mathrm{K}$-gluconate, $20 \mathrm{mM} \mathrm{KCl}, 10 \mathrm{mM}$ Hepes, 2 $\mathrm{mM} \mathrm{MgCl} 2,2 \mathrm{mM} \mathrm{Mg} 2$ ATP, $0.3 \mathrm{mM}$ NaGTP, 7 mM phosphocreatine, and $0.6 \mathrm{mM}$ EGTA ( $\mathrm{pH}=7.2,295 \mathrm{mOsm}$ ). Biocytin was routinely added to patch solution to reveal the morphology of recorded neurons. Immediately after recordings, the slices were fixed in 4\% PFA, and immunostaining for biocytin was performed using streptavidin-Alexa 546-conjugated antibodies as described previously (Khlghatyan et al, 2019).

\section{Data Availability}

The accession number for the gene expression data reported in this article is GEO: GSE137877.

\section{Supplementary Information}

Supplementary Information is available at https://doi.org/10.26508/lsa. 202000707.

\section{Acknowledgements}

This work was supported by funds from Canada First Research Excellence Fund/Medicine by Design, Krembil Foundation, and Canadian Institute for Health Research (CIHR) Foundation grants FDN148455 and FDN143252 to L Attisano and JL Wrana and from CIHR PJT-148568 and Canada Research Chair in Molecular Psychiatry grants to JM Beaulieu.

\section{Author Contributions}

A Sivitilli: conceptualization, formal analysis, validation, investigation, visualization, methodology, and writing-original draft, review, and editing.

JT Gosio: formal analysis, validation, investigation, visualization, methodology, and writing-review and editing.

B Ghoshal: data curation, software, formal analysis, validation, visualization, and writing-review and editing.

A Evstratova: investigation, visualization, methodology, and writing-review and editing.

D Trcka: investigation and methodology.

P Ghiasi: validation, investigation, and writing-review and editing. J) Hernandez: methodology and writing-review and editing.
JM Beaulieu: resources, supervision, funding acquisition, methodology, and writing-review and editing.

JL Wrana: conceptualization, resources, supervision, funding acquisition, and writing-original draft, review, and editing.

L Attisano: conceptualization, resources, supervision, funding acquisition, visualization, project administration, and writing-original draft, review, and editing.

\section{Conflict of Interest Statement}

The authors declare that they have no conflict of interest.

\section{References}

Amin ND, Pasca SP (2018) Building models of brain disorders with threedimensional organoids. Neuron 100: 389-405. doi:10.1016/ j.neuron.2018.10.007

Ayyaz A, Kumar S, Sangiorgi B, Ghoshal B, Gosio J, Ouladan S, Fink M, Barutcu S, Trcka D, Shen J, et al (2019) Single-cell transcriptomes of the regenerating intestine reveal a revival stem cell. Nature 569: 121-125. doi:10.1038/s41586-019-1154-y

Bauwens CL, Peerani R, Niebruegge S, Woodhouse KA, Kumacheva E, Husain M, Zandstra PW (2008) Control of human embryonic stem cell colony and aggregate size heterogeneity influences differentiation trajectories. Stem Cells 26: 2300-2310. doi:10.1634/stemcells.20080183

Becht E, McInnes L, Healy J, Dutertre CA, Kwok IWH, Ng LG, Ginhoux F, Newell EW (2018) Dimensionality reduction for visualizing single-cell data using UMAP. Nat Biotechnol 37: 38-44. doi:10.1038/nbt.4314

Butler A, Hoffman P, Smibert P, Papalexi E, Satija R (2018) Integrating singlecell transcriptomic data across different conditions, technologies, and species. Nat Biotechnol 36: 411-420. doi:10.1038/nbt.4096

Chen HI, Song H, Ming GL (2019) Applications of human brain organoids to clinical problems. Dev Dyn 248: 53-64. doi:10.1002/dvdy.24662

Coucouvanis E, Martin GR (1999) BMP signaling plays a role in visceral endoderm differentiation and cavitation in the early mouse embryo. Development 126: 535-546.

Di Lullo E, Kriegstein AR (2017) The use of brain organoids to investigate neural development and disease. Nat Rev Neurosci 18: 573-584. doi:10.1038/nrn.2017.107

Finak G, McDavid A, Yajima M, Deng J, Gersuk V, Shalek AK, Slichter CK, Miller HW, McElrath MJ, Prlic M, et al (2015) MAST: A flexible statistical framework for assessing transcriptional changes and characterizing heterogeneity in single-cell RNA sequencing data. Genome Biol 16: 278. doi:10.1186/s13059-015-0844-5

Jang HK, Kim BS (2010) Modulation of stem cell differentiation with biomaterials. Int I Stem Cells 3: 80-84. doi:10.15283/ijsc.2010.3.2.80

Kadoshima T, Sakaguchi H, Nakano T, Soen M, Ando S, Eiraku M, Sasai Y (2013) Self-organization of axial polarity, inside-out layer pattern, and species-specific progenitor dynamics in human ES cell-derived neocortex. Proc Natl Acad Sci U S A 110: 20284-20289. doi:10.1073/ pnas. 1315710110

Khlghatyan J, Quintana C, Parent M, Beaulieu JM (2019) High sensitivity mapping of cortical dopamine D2 receptor expressing neurons. Cereb Cortex 29: 3813-3827. doi:10.1093/cercor/bhy261

Khoo ML, McQuade LR, Smith MS, Lees JG, Sidhu KS, Tuch BE (2005) Growth and differentiation of embryoid bodies derived from human embryonic stem cells: Effect of glucose and basic fibroblast growth factor. Biol Reprod 73: 1147-1156. doi:10.1095/biolreprod.104.036673 
Korsunsky I, Fan J, Slowikowski K, Zhang F, Wei K, Baglaenko Y, Brenner M, Loh P-R, Raychaudhuri S (2018) Fast, sensitive, and accurate integration of single cell data with Harmony. BioRxiv. doi:10.1101/461954

Lancaster MA, Knoblich JA (2014a) Generation of cerebral organoids from human pluripotent stem cells. Nat Protoc 9: 2329-2340. doi:10.1038/ nprot.2014.158

Lancaster MA, Knoblich JA (2014b) Organogenesis in a dish: Modeling development and disease using organoid technologies. Science 345: 1247125. doi:10.1126/science. 1247125

Lancaster MA, Renner M, Martin CA, Wenzel D, Bicknell LS, Hurles ME, Homfray T, Penninger JM, Jackson AP, Knoblich JA (2013) Cerebral organoids model human brain development and microcephaly. Nature 501: 373-379. doi:10.1038/nature12517

LaVaute TM, Yoo YD, Pankratz MT, Weick JP, Gerstner JR, Zhang SC (2009) Regulation of neural specification from human embryonic stem cells by BMP and FGF. Stem Cells 27: 1741-1749. doi:10.1002/stem.99

McCarthy DJ, Campbell KR, Lun AT, Wills QF (2017) Scater: Pre-processing, quality control, normalization and visualization of single-cell RNAseq data in R. Bioinformatics 33: 1179-1186. doi:10.1093/ bioinformatics/btw777

Molnar Z, Clowry GJ, Sestan N, Alzu'bi A, Bakken T, Hevner RF, Huppi PS, Kostovic I, Rakic P, Anton ES, et al (2019) New insights into the development of the human cerebral cortex. J Anat 235: 432-451. doi:10.1111/joa.13055

Pettinato G, Wen X, Zhang N (2014) Formation of well-defined embryoid bodies from dissociated human induced pluripotent stem cells using microfabricated cell-repellent microwell arrays. Sci Rep 4: 7402. doi:10.1038/srep07402

Pinto L, Gotz M (2007) Radial glial cell heterogeneity: The source of diverse progeny in the CNS. Prog Neurobiol 83: 2-23. doi:10.1016/ j.pneurobio.2007.02.010

Pollen AA, Bhaduri A, Andrews MG, Nowakowski TJ, Meyerson OS, MostajoRadji MA, Di Lullo E, Alvarado B, Bedolli M, Dougherty ML, et al (2019) Establishing cerebral organoids as models of human-specific brain evolution. Cell 176: 743-756.e17. doi:10.1016/j.cell.2019.01.017

Pollen AA, Nowakowski TJ, Chen J, Retallack H, Sandoval-Espinosa C, Nicholas CR, Shuga J, Liu SJ, Oldham MC, Diaz A, et al (2015) Molecular identity of human outer radial glia during cortical development. Cell 163: 55-67. doi:10.1016/j.cell.2015.09.004
Quadrato G, Brown J, Arlotta P (2016) The promises and challenges of human brain organoids as models of neuropsychiatric disease. Nat Med 22: 1220-1228. doi:10.1038/nm.4214

Quadrato G, Nguyen T, Macosko EZ, Sherwood JL, Min Yang S, Berger DR, Maria N, Scholvin J, Goldman M, Kinney JP, et al (2017) Cell diversity and network dynamics in photosensitive human brain organoids. Nature 545: 48-53. doi:10.1038/nature22047

Rossi G, Manfrin A, Lutolf MP (2018) Progress and potential in organoid research. Nat Rev Genet 19: 671-687. doi:10.1038/s41576-018-0051-9

Sauvageot CM, Stiles CD (2002) Molecular mechanisms controlling cortical gliogenesis. Curr Opin Neurobiol 12: 244-249. doi:10.1016/s09594388(02)00322-7

Stuart T, Butler A, Hoffman P, Hafemeister C, Papalexi E, Mauck WM 3rd, Hao Y, Stoeckius M, Smibert P, Satija R (2019) Comprehensive integration of single-cell data. Cell 177: 1888-1902.e21. doi:10.1016/j.cell.2019.05.031

Vallier L, Alexander M, Pedersen RA (2005) Activin/nodal and FGF pathways cooperate to maintain pluripotency of human embryonic stem cells. J Cell Sci 118: 4495-4509. doi:10.1242/jcs.02553

Velasco S, Kedaigle AJ, Simmons SK, Nash A, Rocha M, Quadrato G, Paulsen B, Nguyen L, Adiconis X, Regev A, et al (2019) Individual brain organoids reproducibly form cell diversity of the human cerebral cortex. Nature 570: 523-527. doi:10.1038/s41586-019-1289-x

Wamsley B, Fishell G (2017) Genetic and activity-dependent mechanisms underlying interneuron diversity. Nat Rev Neurosci 18: 299-309. doi:10.1038/nrn.2017.30

Watanabe M, Buth JE, Vishlaghi N, de la Torre-Ubieta L, Taxidis J, Khakh BS, Coppola G, Pearson CA, Yamauchi K, Gong D, et al (2017) Self-organized cerebral organoids with human-specific features predict effective drugs to Combat Zika virus infection. Cell Rep 21: 517-532. doi:10.1016/ j.celrep.2017.09.047

Yoon S), Elahi LS, Pasca AM, Marton RM, Gordon A, Revah O, Miura Y, Walczak EM, Holdgate GM, Fan HC, et al (2019) Reliability of human cortical organoid generation. Nat Methods 16: 75-78. doi:10.1038/s41592-0180255-0

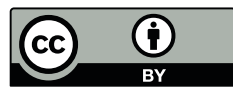

License: This article is available under a Creative Commons License (Attribution 4.0 International, as described at https://creativecommons.org/ licenses/by/4.0/). 